\title{
The Innovative Development of News Dissemination in a Media Convergence Environment--Taking Kanghui VLOG as an Example
}

\author{
Liu Yudian ${ }^{1}$, Li Chenhao ${ }^{2, a^{*}}$, Guo Yixin ${ }^{3}$ \\ ${ }^{1}$ School of Journalism, Communication University of China, Beijing, 100024 \\ ${ }^{2}$ School of Economics and Management, Beijing University Of Technology, Beijing, 100124 \\ ${ }^{3}$ Advertising Institute, Communication University of China, Beijing, 100024 \\ aEmail: lichenhao0913@163.com
}

\begin{abstract}
With the development of science and technology, the media industry is undergoing extensive and profound changes. In order to cater to the needs of the audience, traditional media are also exploring convergence along with its development, trying to present traditional news reports in a more lively and vivid way through various platforms. Traditional news has gone through transformation and upgrading both in its form and content. In November 2019, CCTV (China Central Television) News Channel released "Kanghui VLOG" on Weibo, Bilibili and other platforms, which embodies the transformation and upgrading of traditional news communication in the context of media convergence. This article will take "Kanghui VLOG" as the main object of study, focusing on news content and features, and exploring what efforts have been made by traditional news for the transformation and upgrading and its significance of in the process of media convergence.
\end{abstract}

Keywords: media convergence, news communication, VLOG, mainstream media, traditional news.

\section{KANGHUI VLOG BACKGROUND}

\subsection{The era background of media convergence}

In 1978, Nicholas Negolo Ponti, the founder of the Massachusetts Institute of Technology's Media Lab, predicted that "all communication technologies are suffering from joint deformation"; "The three fields of broadcasting and animation industry, computer industry, printing and publishing industry will tend to merge", etc.

In the 21 st century, with the development of technology, the media industry, telecommunications industry, and IT industry are all competing on a global scale, and various fields are constantly trending toward the convergence of media. "Media convergence " is the inevitable evolution trend of the media industry under digital technology. It is a new form of information production that takes the media industry as the core, and multiple industries such as information production, information dissemination, and information application use "fusion" as the production method.
The same is true for the news media industry. Due to changes in production methods and communication methods, more audiences gather on new media platforms, such as Weibo, which has more than 500 million monthly active users. To a certain extent, mainstream media with declining discourse power began to explore new development paths, hoping to break the usual serious image for news dissemination, reach and guide audiences in a more approachable way, upgrade news content and format, and enable traditional mainstream media adapt to the new media environment.

\subsection{The rise of VLOG as a format of short video}

VLOG is a type of web diary, its full name is VIDEO BLOG, or video blog. The photographed works use the form of video instead of the simple photo-text shooting $\log$, which has a strong timeliness, and this feature is also quite similar to the news that pursues timeliness. In addition, VLOG also has the characteristics of lifelike, personalized and entertaining.

In 2012, uploader on the Youtube platform released the first VLOG. With the development of Internet 
technology and the progress of globalization, VLOG began to gradually enter Chinese market in 2018. With the increasing number of VLOG photographers on a wide range of new media platforms, and the promotion of some star VLOG, VLOG first became known to a wider public, then become more and more widespread.

\section{CONTENT AND FEATURES OF "KANGHUI VLOG"}

\subsection{Content}

On November 10, 2019, CCTV News uploaded the first Kanghui VLOG "Kanghui's First Vlog: It's Time to Go Far" on Weibo, Bilibili and other platforms. From November 10 to November 15, the first season of Kang Hui's VLOG series uploaded seven videos, each of which lasted about two minutes. The main content was Kang Hui's follow-up reports of President Xi Jinping's state visit to Greece and the attendance to Brazil for the 11th BRICS Leaders' Meeting.

In the first video, "Kang Hui's First Vlog: It's Time to Go Far", Kang Hui showed the official passport used for follow-up visits to the camera at CCTV, showed the suitcase he was going to carry, and briefly introduced the follow-up filming team together with him.

The second video is "Kang Hui's second Vlog: What is the first thing I did after arriving in Athens and getting off the plane". When Kang Hui arrived in Athens, Greece, he arrived at the airport, met with colleagues in the airport media area at once. He took the picture of General Secretary Xi Jinping and Peng Liyuan arriving in Greece and getting off the plane. Kang Hui described in the video that this was the second state visit of the Chinese President to Greece after a lapse of eleven years. At the same time, Kang Hui also showed an article about President Xi Jinping in the Greek "Daily".

The third video "The third Vlog is here! Kang Hui's vlog was also snatched" tells the scene of Kang Hui and other staff arriving at the Greek Presidential Palace for interviews and reports, and filmed the leaders' meeting at the welcoming ceremony and pictured the Greek Presidential Guard.

In the fourth video "Kang Hui's vlog is updated again!" He self-deprecated himself as a Selfie Rookie", Kang Hui arrived at the port of Piraeus and talked about the port situation where the Greek Prime Minister accompanied President Xi Jinping to inspect the port. Kang Hui also interviewed Xu Lirong, chairman of China Ocean Shipping Group, in this VLOG, but due to poor acoustic reception, Kang Hui also complained that he was a self-portrait rookie.

The fifth video of "Kanghui vlog does not delay! Kang Hui Reveals the Secret Weapons in the Vlog" filmed the scene of Kang Hui and the follow-up press team arriving in Brasilia, the capital of Brazil, and demonstrated the news scene where the leaders of the five BRIC countries gathered to focus on scientific and technological innovation cooperation to promote BRIC cooperation. In addition, Kang Hui also revealed the secret weapon -- fill light, which was mentioned in the first VLOG in this video, responding to the concerns of netizens.

The sixth VLOG is "Kang Hui vlog: What does Kang Hui's workshop look like in Brazil? " The main content is that after the closing ceremony of the BRICS Business Forum was held, Kang Hui led the audience to experience the customs of Brasilia and visited the meeting room of 11th BRICS. In addition, he also showed the audience his studio, with the unique and clean workshop as well as dubbing pictures with pillows for soundproof.

The seventh VLOG is " first season of Kang Hui's vlog ends! Mysterious Guests on screen". Kang Hui filmed the eleventh meeting process of the leaders of the BRICS countries in the Ministry of Foreign Affairs of Brazil, and also showed the media colleagues on the front lines of major country diplomacy.

\subsection{Features}

\subsubsection{In Aspect of Form}

\subsubsection{Diversified media}

First of all, "Kanghui VLOG" has various media for communication. Compared with traditional news broadcast on TV, "Kanghui VLOG" is released on multiple new media platforms, including Weibo, Bilibili horizontal-format video, and vertical-format video of CCTV news accounts at Tiktok. Due to the different audience groups of various platforms, the release of videos by multiple media has increased the amount of video playback and increased the attention of news.

In addition, when "Kanghui VLOG" was launched on different platforms, it did not forget to cater to the habit of the audiences, watching videos on various platforms, and adjusted the horizontal and vertical videos to meet the diverse needs of viewers.

\subsubsection{Unique dissemination perspective}

VLOG refers to video log, which is a form of video recording toward life that is from a personal perspective. The same is true for "Kanghui VLOG". The host who always carrying a serious image becomes a vlogger who raises the camera to take selfies. The presentation of perspective is very different from traditional current affairs news. Traditional news tells recent facts from a more objective and comprehensive perspective. However, "Kang Hui VLOG" takes Kang Hui as the main character. From his personal perspective, it presents stories from 
subjective and more limited perspectives, showing a more easy-going image of news anchors and CCTV news.

Therefore, the audience is more likely to bring their subjective perspective and feel more involved in news events. News also weakens the previous sense of authority and solemnity for the audience, making it more approachable for the audience to more easily accept and understand. For example, on the Bilibili platform, the most popular comment to one of Kanghui's VLOG indicates that "CCTV has followed the popular trend of a down-to-earth hosting style in recent years. It is really great, whether it is a vlog or talking about simulcasting by the anchor and so on, they all have a strong interaction with our people. The manner of a big country is to go out and tackle domestic affairs with a easy grace. Mr.Kang is both interesting and confident in front of the camera. I feel that I am also standing at the front line of diplomacy."

\subsubsection{High interactivity}

At the same time, CCTV News is also actively interacting with the audience of "Kanghui VLOG" on different platforms. For example, on the Weibo platform, the topic \#Kanghui's vlog\# has so far received 132,000 comments and 250 million views. CCTV News also pays attention to topic leading and encourages audiences to discuss about "Kanghui VLOG", creating topics such as \#Kanghui 's pronunciation is right in this VLOG?\#, \#Kanghui self-deprecated himself as a Selfie Rookie\# and other themes.

CCTV News has grasped the open and interactive characteristics of these platforms as self-media platforms. Those are different from the relatively single traditional media that only outputs traditional news content to the audience. Such new media actively interact with the audience and give likes to the comments of the audience. Meanwhile, they alleviates the oppressing sensation brought by traditional news media, and penetrates into the lives of new media audiences.

\subsubsection{In Aspect of Content}

\subsubsection{High video quality}

First of all, "Kanghui VLOG" is rich in content. Although each VLOG only lasts about two minutes, it covers a wide range of content, including both news frontline scenes, natural sightseeing and cultural scenes. It also does not forget to continuously output mainstream values, demonstrate news facts, and tell the story of China.

Secondly, the content of "Kanghui VLOG" is extremely unique. Because it is CCTV news official production, the level is certainly higher than the VLOG that new media audiences usually watch. "Kanghui VLOG" shows the frontiers of China's diplomacy, such as the group photo of the leaders of the five BRICS countries, etc. Therefore, many viewers commented on
"Kanghui VLOG" that they could see the real-life "Uncle $\mathrm{Xi}$ and Aunt Peng" and felt these news works very "hardcore". . At the same time, "Kanghui VLOG" shows the daily work of CCTV journalists, including their "business passport", "news anchor recording studio", "Kanghui suitcase", etc., which audiences usually don't have the opportunity to touch. Therefore, "Kanghui VLOG" is not only different from the VLOG shot by ordinary self-media, but also has many new features compared with the audience's daily life, which satisfies the audience's curiosity.

In addition, "Kanghui VLOG" is also good at creating suspense, which can enhance the sense of interaction among the audiences. For example, in the first issue of VLOG, Kang Hui seeded about "fill flash", and it was not announced to the audience until the fifth issue; in the first issue of VLOG, Kang Hui failed to pronounce the word VLOG. The audience pointed out and Kang corrected it in the second issue. He said "Read it right this time?", which narrowed the distance with the audience.

\subsubsection{Lively video style}

The shooting style and editing style of "Kanghui VLOG" are very lively, which fits perfectly with the presentation of new media videos. When filming videos, Kang Hui spends most of his time taking selfies, using buzzwords to express, calm but lively. Even when interviewing $\mathrm{Xu}$ Lirong, chairman of China COSCO Shipping Group, Kang Hui held a camera to take a selfie, which is very different from the traditional interview style of journalists.

In addition, "Kanghui VLOG" is also equipped with a large number of gay music and flexible transitions. The overall video style is lively and bright but does not lack the promotion of the main theme. It successfully caters to the audience's aesthetic needs.

\section{THE SIGNIFICANCE AND INFLUENCE OF "KANGHUI VLOG"}

"Kanghui VLOG" is a very positive attempt. CCTV News releases new media short videos with the theme of "Forefront of Great Power Diplomacy" and VLOG as the form on new media platforms, reducing the seriousness of traditional news dissemination and bringing it closer to audiences. It successfully won the love of a large number of followers. "Anchor Talking News Series", which is similar to "Kanghui VLOG", also has received similar good reputation. There are even viewers who urged more works like "SaBeining VLOG", "CCTV Boys" and so on.

"Kanghui VLOG" also created a brand-new news reporting method-- "VLOG News". Traditional news focuses on macro-narratives, with procedural reporting and less innovation. It is not attractive to audiences in the era of integrated media, especially toward young 
audiences, and cannot actively and effectively disseminate news information. "Kanghui VLOG" allows mainstream media to better fit into the audience, advance public opinion guiding, reconstruct the discourse power in the new media, increase influence, and broaden the channels of expression and transmission of traditional news.

Ordinary self-media have a slightly narrower scape of view. The videos uploaded by VLOG creators on various platforms generally share their own lives, mainly about traveling, work and study, etc., and have less guiding of mainstream values. The new mainstream media strives to guide mainstream values, but because the content is boring and the way of presentation is not approachable, it is difficult for the audience to accept, and it is easy to produce adversarial decoding. Therefore, "Kanghui VLOG" is close to the life of the audience, responds to the audience's attention, and softens the hard news. For example, in the port of Piraeus after the inspection of President Xi Jinping, Kang Hui introduced the Greek folk song "Children of Piraeus". He stood among the Chinese and Greek staff working in the port of Piraeus and said that " I firmly believe that the children of Piraeus will have a better tomorrow, and in the future, there will be more touching Chinese stories in the singing of the Greek people." In addition, General Secretary Xi Jinping delivered an important speech at the BRICS Leaders' Meeting, pointing out that BRICS cooperation "has a close tie in history and a promising future." Kang Hui in the BRICS meeting in the VLOG back on to the flags of the five countries, explaining that "China's development is an opportunity for the world. This has been fully verified in the first ten years of BRICS cooperation. At the beginning of the second golden decade, we also believe that China will continue to add color to this "brick" by opening up and innovative actions." Through the form of VLOG, traditional news better conveys political discourse, expresses China's diplomatic information to the audience, and shows the country's friendly image of diplomacy as a major country.

\section{CONCLUSION}

In short, "Kanghui VLOG" is an active and successful exploration of traditional news dissemination within the environment of media convergence. It dispelled the original serious image, enriched the content of the report, narrowed the distance with the audience, and promoted the leading of public opinion, expanded the influence of mainstream media. It is more conducive to the innovative development of the transformation and upgrading of mainstream traditional media in the era of media convergence in the future.

\section{REFERENCES}

[1] Zhu Chunyang, Liu Xinyi, Yang Hai. How to shape the new mainstream media and modern communication system in the era of media convergence[J]. Journalism University, 2014(6).

[2] He Qian. Analysis of "Kang Hui Vlog" Weibo Communication from the Perspective of Media Convergence [G]. School of Journalism and Communication, Nanning Normal University, 2020(10).

[3] Feng Ziwei. Exploring the innovative presentation of foreign affairs news in the new mainstream mediataking Kang Hui Vlog as an example [G]. School of Journalism and Information Communication, Huazhong University of Science and Technology, 2020 (09).

[4] Gao Shufan. The new characteristics of current political news under the environment of integrated media-take "Kang Hui's Vlog" as an example [G]. Hebei Normal University, 2020 (10). 